\title{
Deep Learning Neural Networks as a component of a model of saccadic generation
}

\author{
Sofia Krasovskaya \\ School of Psychology \\ Vision modelling Lab \\ HSE University \\ Svkrasovskaya@hse.ru
}

\author{
Georgii Zhulikov \\ Vision modelling Lab \\ HSE University
}

\author{
W. Joseph MacInnes \\ School of Psychology \\ Vision modelling Lab \\ HSE University
}

\begin{abstract}
Approximately twenty years ago, Laurent Itti and Christof Koch created a model of saliency in visual attention in an attempt to recreate the work of biological pyramidal neurons by mimicking neurons with centre-surround receptive fields. The Saliency Model has launched many studies that contributed to the understanding of layers of vision and the sphere of visual attention. The aim of the current study is to improve this model by using an artificial neural network as the spatial component of a model that generates saccades similar to how humans make saccadic eye movements. The proposed model uses a Leaky Integrate-and-Fire layer for temporal predictions, and replaces parallel feature maps with a deep learning neural network in order to create a generative model that is precise for both spatial and temporal shifts of attention. Our model was able to predict eye movements based on unsupervised learning from raw image input, combined with supervised learning from fixation maps retrieved during an eye-tracking experiment. The results imply that it is possible to match the spatial and temporal distributions of the model to spatial and temporal human distributions.
\end{abstract}

Keywords: Itti and Koch, saliency models, deep learning neural networks, leaky integrateand-fire, visual attention, saccadic generation 


\section{Introduction}

Contemporary cognitive science may be viewed as a product of the cognitive revolution, which embraced a tendency to use an interdisciplinary and computational approach in research in order to gain a better understanding of cognitive processes. One of the main methods in this approach is computational modelling, which is based on the notion that through the creation and investigation of artificial models of mental processes it is possible grasp the complex notion of human cognition in a more precise way (Broadbent, 1987). Based on this approach, it is possible to apply existing tools to theory in order to address human cognition holistically. One of the applications of computer modelling in cognitive psychology is to simulate human vision processing. Some of the best approaches to computational modelling focus on both the biological and theoretical aspects of vision, such as the classical Itti and Koch salience model (Figure 1) (Itti \& Koch, 2000). This model combines biological plausibility, by implementing pyramidal cells that work like receptive fields of the visual cortex, and the feature integration theory of attention (Treisman \& Gelade, 1980), by implementing multiple pre-attentive feature processing streams. Such models were novel in their time in terms of spatial distribution accuracy, but they were surpassed in terms of temporal accuracy by other approaches, such as diffusion models (MacInnes, 2017).

In addition to modelling spatial salience, temporal models may predict the time course distribution of human saccadic latencies. The classic Itti and Koch integrated this temporal prediction in addition to image processing, but many recent solutions do not. The most prominent examples of such models of temporal generation are the 'Leaky integrate-and-fire' (LIF) (Maass, 1997), the leaky competing acumulator (Usher \& McClelland, 2001) and the drift diffusion algorithms (Ratcliff, 1978; Ratcliff \& McKoon, 2008). The LIF, otherwise called a spiking network, is similar to a biological neural network. Just like biological neurons fire upon reaching a threshold, the spiking network uses accumulation of evidence to gain a certain amount of information from an incoming signal and pass this information once it receives enough input. A distinctive feature of such neurons is that the accumulated data 'leaks' when the input signal ceases.

In general, there are many approaches with advantages and disadvantages based on the field of application and the potential tasks. However, we believe that in order to properly model human saccadic eye movements it is important to have a balanced combination of all the advantages of the existing models, which supposes good temporal and spatial characteristics, as well as high biological plausibility. In an attempt to find this balanced 
approach, we have decided to implement deep learning neural networks as a component of our model to investigate the visual system.

\section{Deep Learning: a tool for modelling cognitive processes}

Deep learning algorithms have proven useful in different spheres, such as biology, chemistry, finances, business, physics and neuroscience (Basheer \& Hajmeer, 2000). These algorithms are a versatile, accurate and powerful tool, which has been acknowledged and proven by recent studies in object and speech recognition, as well as in other domains linked to complex data analysis (LeCun, Bengio \& Hinton, 2015).

In the field of visual research and eye-movement prediction, deep neural networks have become the leaders among other approaches. For instance, there is a tendency to choose more complex deep hierarchical structures over the Itti and Koch (2000) salience model (Bylinskii et al., 2016; Judd, Durand \& Torralba, 2012). The reason for this is due to the fact that deep learning algorithms are effective tools for modelling high levels of abstraction (Bengio, 2009), such as image and auditory stimuli. However, there are limitations to these successes. Artificial models tend to have difficulties predicting fixations based on semantic content since people tend to look at points in space that are meaningful within a specific context. The fact that models are bad at such semantic-related predictions is called the semantic gap. Deep learning models also may provide a possibility to model human visual attention more accurately by narrowing the semantic gap between model and human predictions (Huang et al., 2015).

\section{Modelling in human vision}

More recent models, however, focus primarily on the spatial accuracy of the predictions (Bylinskii et al., 2016). Even models that do consider temporal accuracy, the reaction times tend to be too fast as compared to human reactions, which leads to poor matches of temporal distributions in such models (Merzon et al, 2020). In addition, the LIF often used in such models do not contain inherent noise so it is not possible to judge the RT distribution of such a model without aggregating data on many pictures. This way the resulting variance in the distribution solely depends on the pictures themselves and not on the model. Contrasted to that, human distributions tend to be log normal even on a single picture (Figure 2) (MacInnes, 2017; Mezon et al., 2019). There are models that better match human reaction times, such as diffusion models (MacInnes, 2017), or leaky 
neuron models (Usher \& McClelland, 2001) but these models tend to abstract space to achieve that temporal performance.

Since the aim of this research was the creation of an artificial neural network that would be able to learn to generate human-like saccades, it was important to choose an algorithm that would mimic this generative nature of the visual system. For this it was necessary to take into account both the spatial and the temporal aspects of the process. Therefore, a combination of deep learning algorithms and an LIF component were chosen as the main components of the model. The Deep learning neural nets have been shown to be extremely accurate in predicting spatial salience maps, as seen on the MIT Benchmark website (Bylinskii et al., 2015), and we used this approach to create a spatial salience map. This map was further processed by a LIF layer to produce the final predictions in space and time. Despite its limitations, the LIF algorithm was chosen over diffusion (MacInnes, 2017; Ratcliff \& McKoon, 2008), accumulator (Usher \& McClelland, 2001) and race (Purcell et al., 2012; Wolfe, 1994; Wolfe \& Gancarz, 1997) models primarily since LIF models have accurate 2D spatial representations of visual space and are still best for natural scene processing.

\section{Methods}

Our model used the Matlab Neural Network toolbox (https://www.mathworks.com/products/neuralnetwork.html) and implemented a combination of stacked autoencoders leading to a softmax layer. This model included two layers of autoencoders which accepted as input images of real indoor scenes with dimensions of 1024x768. Output from the softmax layer were fixation maps with dimensions $33 \times 50$ per image. Initial training of the autoencoders used unsupervised learning of network weights using images alone preceding a supervised learning phase where all layers, including the softmax layer was further optimized. The dataset consisted of 59 training images and 18 with testing and training for each of 35 participants, which made a total of 1115 training maps and 478 testing maps. The images with a 4:3 ratio were taken from the LabelMe dataset (Russell et al., 2008).

Step 1 - preprocessing. Each image was proportionally rescaled to a width of 120 and a height of 90 and reshaped from a matrix into a vector array of values between 0-255. Due to the fact that many salient points never reached figures higher than 100, the values were normalized to a distribution of values between 0 and 1 
in order for these points to be vivid on the map. Each image was paired with matching fixation maps and their order was randomised.

Step 2 - unsupervised early feature extraction. The processed training images were used as input to the first autoencoder layer of the model. The training parameters of this layer were set to 200 iterations and hidden layers to size of 50. The learned hidden features were then extracted and saved as a separate variable.

Step 3 - unsupervised late feature extraction. The extracted features from layer 1 were passed on to the second autoencoder layer for further training. The parameters of this layer were set to 200 iterations and a hidden size of 50. The learned hidden features were then extracted and saved as a separate variable.

Step 4 - supervised salience training. The extracted features from the second layer together with the processed training fixation maps were used to train the softmax layer. The parameters were set to default with 100 iterations.

Step 5 - supervised Deep training. The autoencoder layers and the softmax layer were stacked together to form a deep network. The stacked network was trained on the training images and training maps. The trained deep network with all its parameters and values was then saved as a separate file for convenience during the testing phase.

Step 6 - testing. The network was tested on the testing data. The predictions were reshaped to a dimension of $33 \times 50$ and normalised. This step was important in order to normalise and smoothen all matrix values between 0 and 1 , otherwise the highest and lowest values were unevenly spread between 0 and 255 , with the lower values tending to zero and the higher values tending to 255 , which lead to a highly contrasted map.

Step 7 - validation. The network was tested on the testing data. The predictions were reshaped to a dimension of $33 \times 50$ and normalised using the abovementioned formula.

Step 8. For visual assessment, resulting salience maps were plotted in pairs of training maps against predictions based on the training data and testing maps against predictions based on the testing data.

Step 9. All 77 images together with the predicted fixation maps were passed on to the Leaky integrateand-fire layer created in the Saliency Toolbox (Walther \& Koch, 2006) which uses a 'winner take all' algorithm to produce a reaction time. The temporal limit was set to 2 seconds and the parameters were set to default and are listed in Table 2. 


\section{Metrics for result analysis}

In order to evaluate the performance of the saliency algorithm it was necessary to implement certain evaluation metrics. As the saliency part of the network dealt with spatial locations, the reasonable choice was to use a location-based metric algorithm in order to assess the performance of the generated saliency maps. One characteristic instrument of such metrics is the area under the Receiver Operating Characteristic curve (AUC ROC), which estimates the tradeoff between true positive and false positive values at different discrimination thresholds by verifying that true positives are labelled before negative values (Ferri, Hernández-Orallo \& Flach, 2011; Bylinskii et al., 2016). The AUC unit of measurement is one of the most widely used instruments for saliency assessment (Judd, Durand, \& Torralba, 2012), as shown on the MIT saliency benchmark website (Bylinskii et al., 2015).

The specific AUC metric that was chosen for the evaluation task was the AUC-Judd (Judd, Durand, \& Torralba, 2012; Riche, Duvinage, Mancas, Gosselin \& Dutoit, 2013). The AUC-Judd interprets fixations as a classification task, where a pixel of the map may be either salient or not by applying a threshold over the intensity value of the saliency map (Kachurka et al. 2015). Each salient pixel against human fixations on the map is considered a true positive value, whereas salient pixels over non-fixation areas are classified as false positive values. The final AUC score is then calculated and plotted as a tradeoff between true and false positive values. The highest possible score may be 1 , whereas a 0.5 score is considered as random performance. Thus, any score lower than 0.5 is regarded as unsatisfactory.

Our proposed saliency model has proven to have an above average performance on the AUC Judd test. The average score on the test for 18 generated maps was estimated as 0.61 , which is almost equal to the score of the first model proposed by Dirk Walther and Christof Koch at the MIT saliency benchmark website (Walther $\&$ Koch, 2006), with the lowest score among the maps equal to 0.54 (figure 5) and the highest score equal to 0.70 (figure 6). Although these results do not match the best accuracy from newer DLNN's like Deep Gaze $($ AUC-JUDD $=0.88)($ Kümmerer et al, 2017), our model goes beyond being a basic a classifier of spatial salience. Like the older Salience solutions of Itti \& Koch, our solution is a temporal, generative model of fixation selection. 


\section{Results and conclusion}

The approach proved to be efficient in terms of spatial accuracy. The model predicted fixation maps similar to human fixation maps. As seen in figure 3, the model produced realistic maps based on full set of human fixation maps per image.

The current results demonstrate that the deep-learning model based on the Itti and Koch saliency model (Itti \& Koch, 2000) is effective in generating salience maps of visual space.

In terms of temporal accuracy, however, the leaky integrate-and-fire algorithm did not entirely meet our expectations. Despite addressing, theoretically, both spatial and temporal issues of modelling saccadic eye movements, the classic LIF approach with default parameters has proven to have limitations in terms of temporal biological plausibility. The model does not produce a distribution by itself. It only mimics distributions based on differences between images in the data set. Results have shown good findings with regard to the mean and z-test, however, the overall distribution has proved the approach to be ineffective regarding biological accuracy (Merzon et al, 2020). The reaction times produced by the model fit into the range of human saccadic reaction times; however, they seem to have a deterministic nature when used with default parameters. Besides, the bimodality in the distribution was present throughout all testing phases (figure 4).

The current study has demonstrated that it is possible to improve classical theories with the help of new tools, such as deep learning neural networks. Just like Itti \& Koch's salience model (Itti and Koch, 2000) showed how pyramidal neurons work, the described model is an attempt to partially visualize how the superior colliculus works by using 'accumulation of evidence' modelling. Each layer of the network makes different contributions to the model, just like the human visual system processes visual information, which provides a good foundation for future research. However, it is necessary to further investigate the temporal aspect as well as to focus on the frames of reference in the human visual system in order to improve spatial predictions of the model in a physiologically accurate manner.

\section{Acknowledgments}

This work is partially supported by the HSE academic fund program for the scientific research lab "Vision Modelling Lab". 


\section{Figures and Tables}

(a)

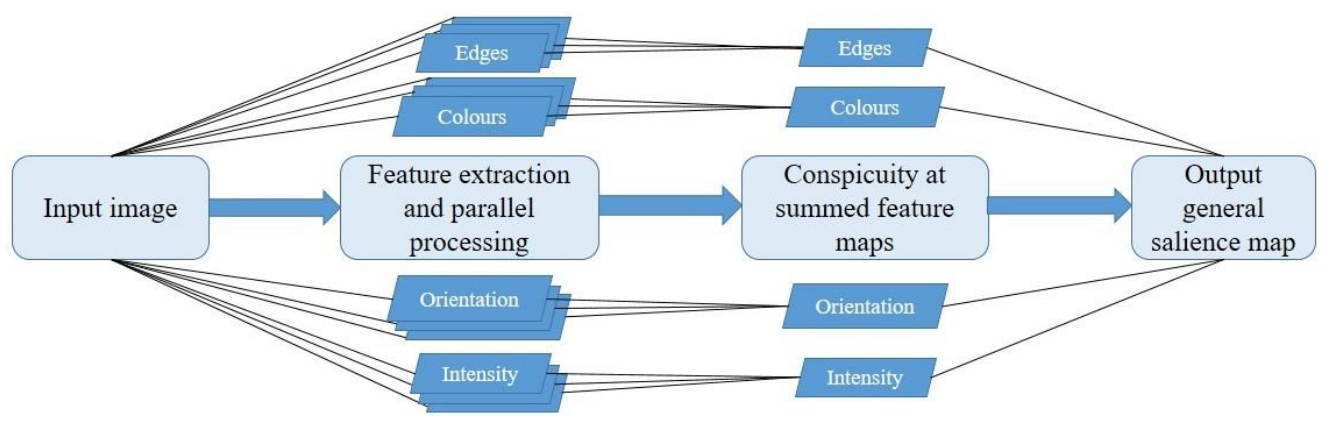

(b)

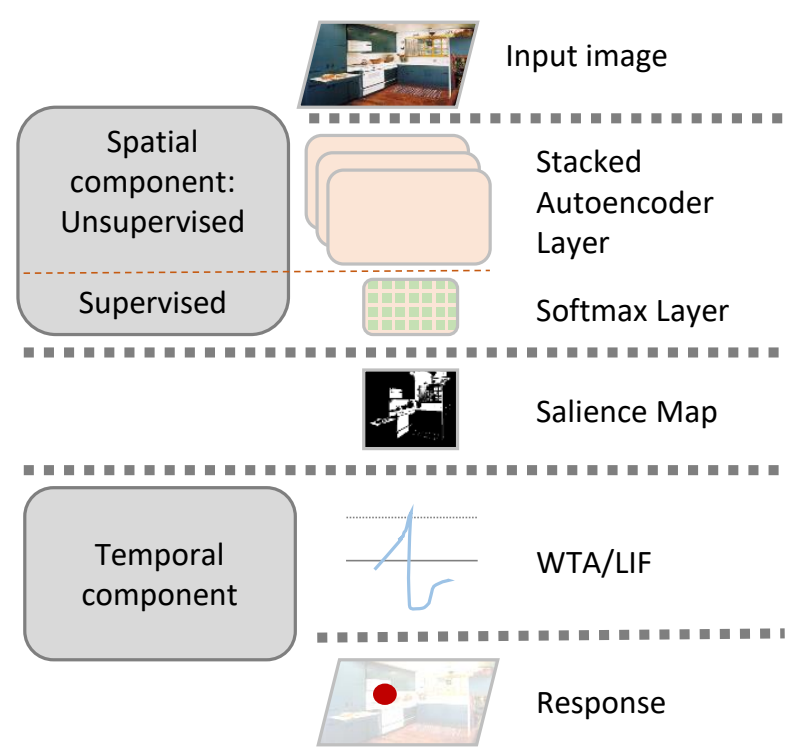

Figure 1. (a) A generalized demonstration of the salience computation algorithm present in many existing models based on the original Itti and Koch salience model (Itti \& Koch, 2000), which predicted both where and when shifts of attention would occur for a given image input. From "Salience Models: A Computational Cognitive Neuroscience Review," by S. Krasovskaya and W. J. MacInnes, 2019, Vision, 3(4), 56 (https://doi.org/10.3390/vision3040056) and (b) our implementation. From "Temporal Limitations of the Standard Leaky Integrate and Fire Model," by L. Merzon, T. Malevich, G. Zhulikov, S. Krasovskaya and W. J. MacInnes, 2020, Brain Sciences, 10(1), 16 (https://doi.org/10.3390/brainsci10010016) 
A. Latency of the first saccades per image

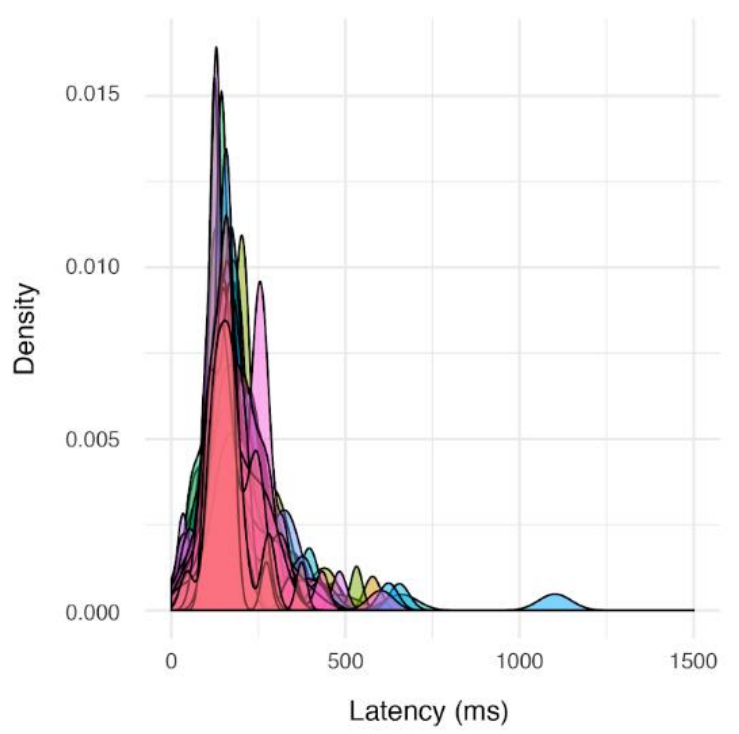

B. Latency of all saccades per image

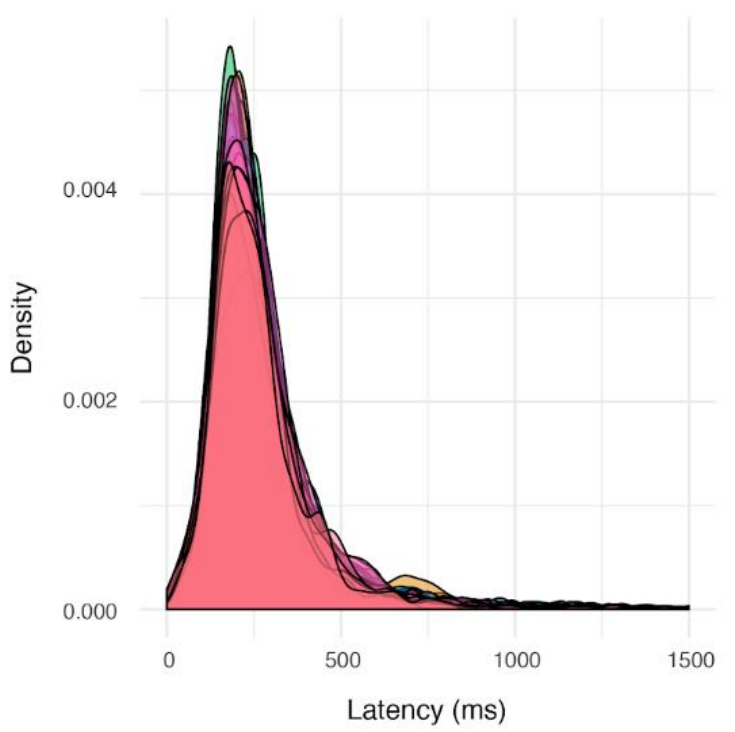

Figure 2. A demonstration of the bad matching using the default parameters of the Itti and Koch (2000) salience model with a LIF layer to human saccade latency data (MacInnes, 2017). 'Density' corresponds to the proportion of eye movements made in the time period. 


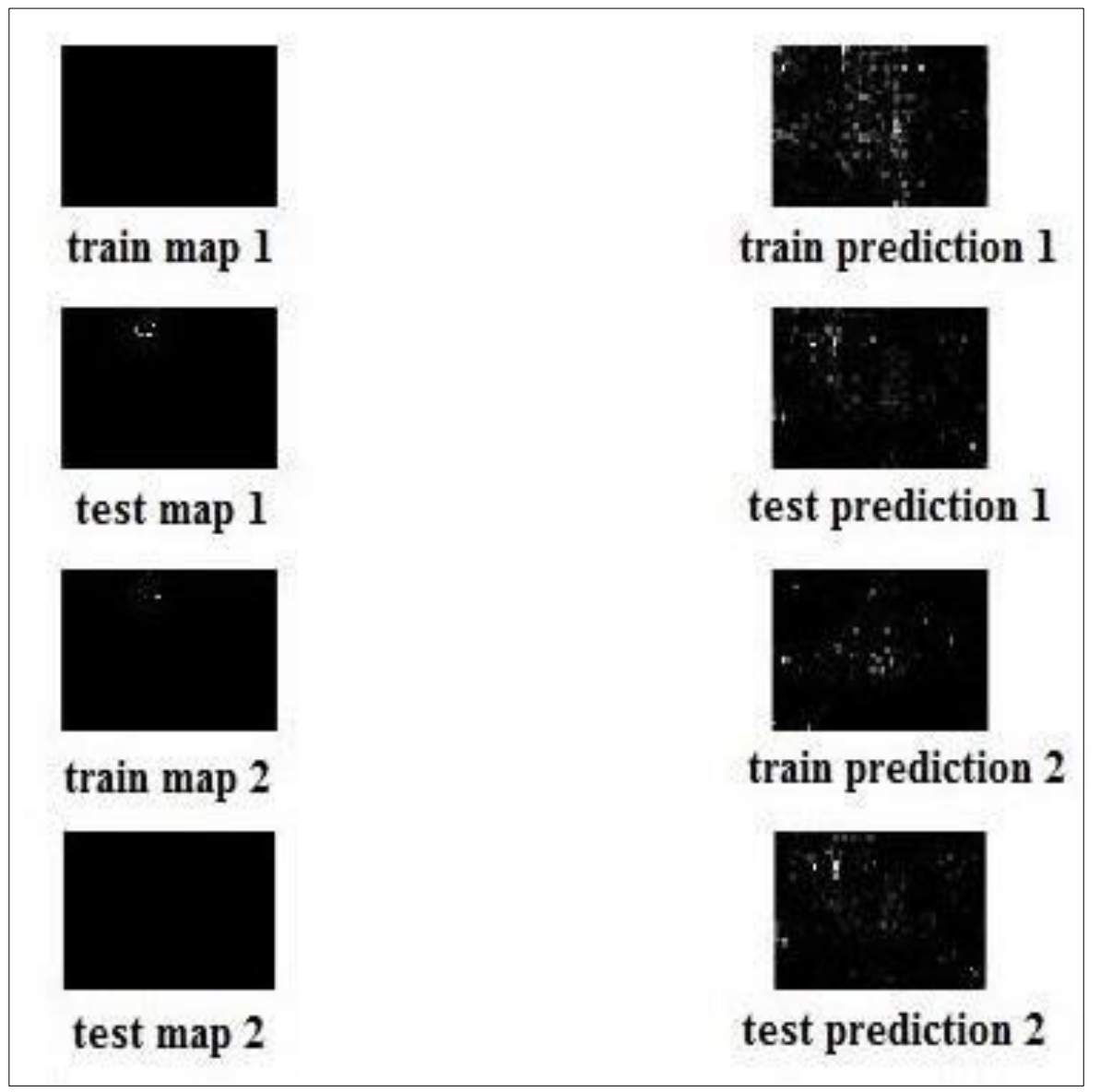

Figure 3. A demonstration of the autoencoder+softmax model spatial predictions. 

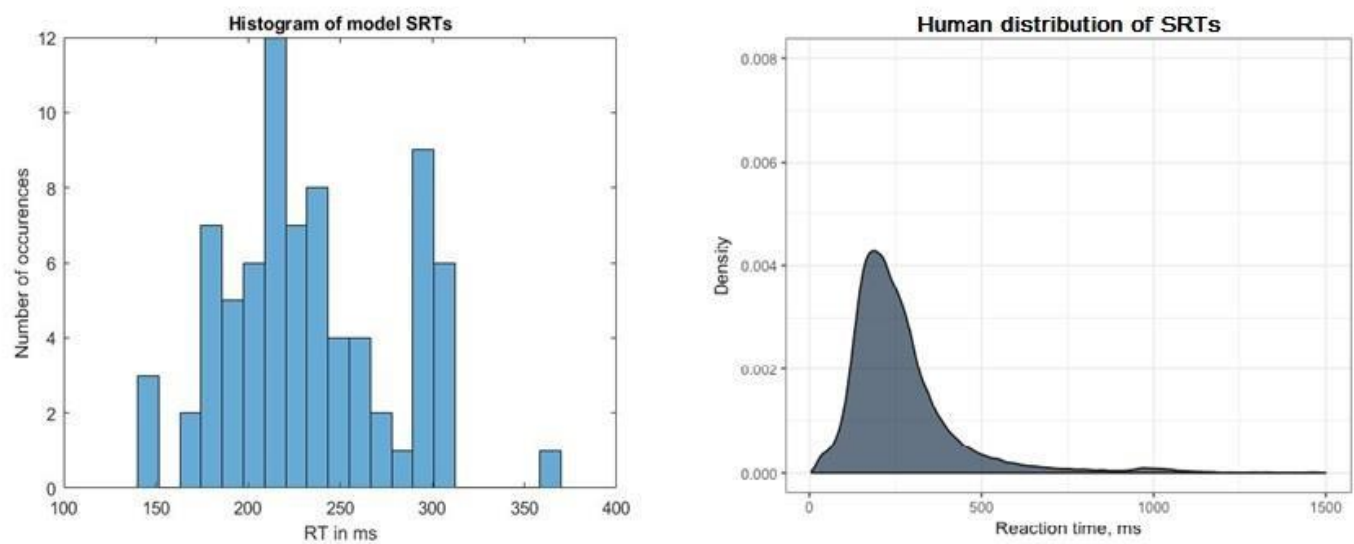

Figure 4. Distribution of SRTs produced by the model (left) against human distribution (right). 


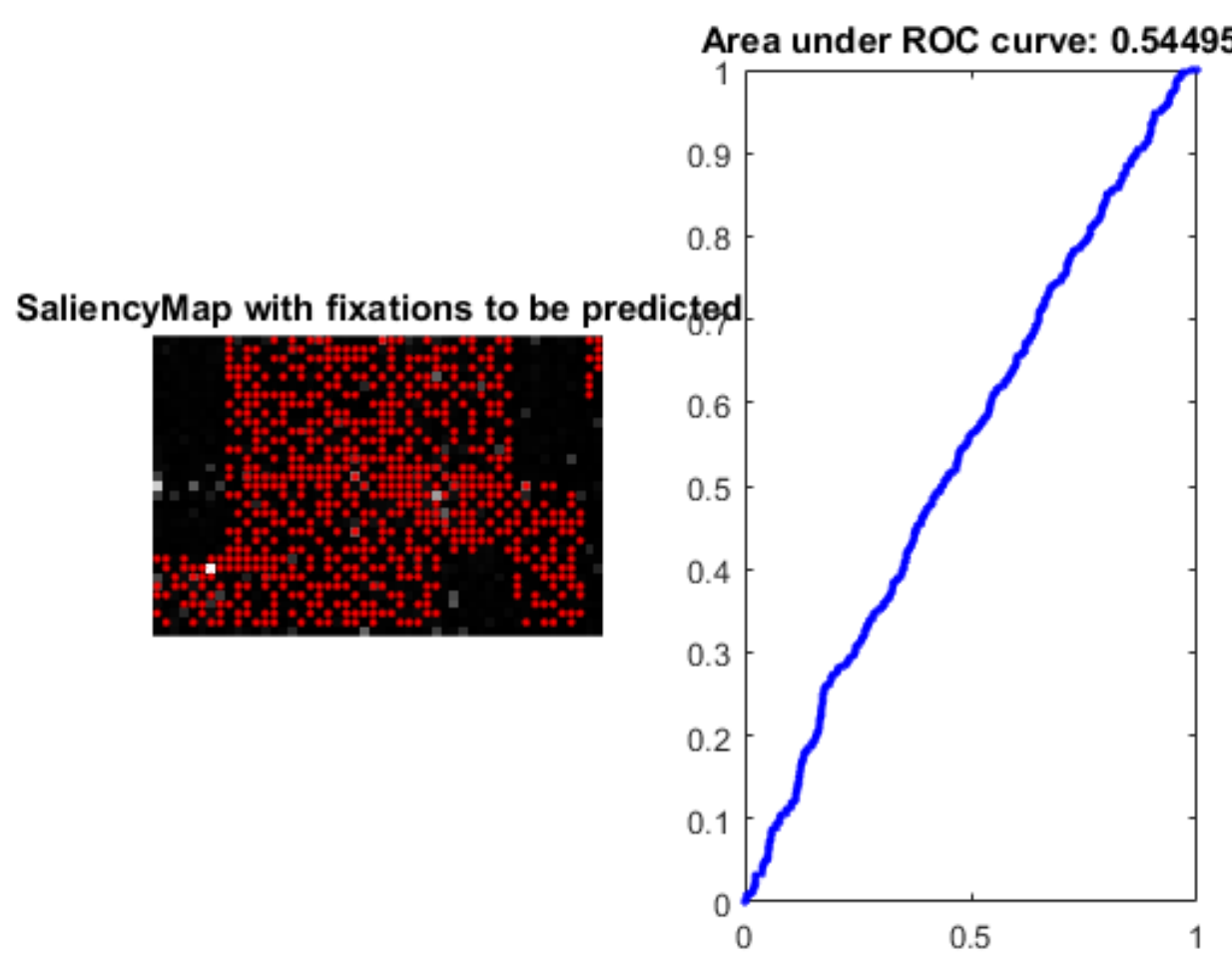

Figure 5. The minimal score of the generated maps on the AUC-Judd test. FP are the False Positive variables, TP - the true positive variables. 


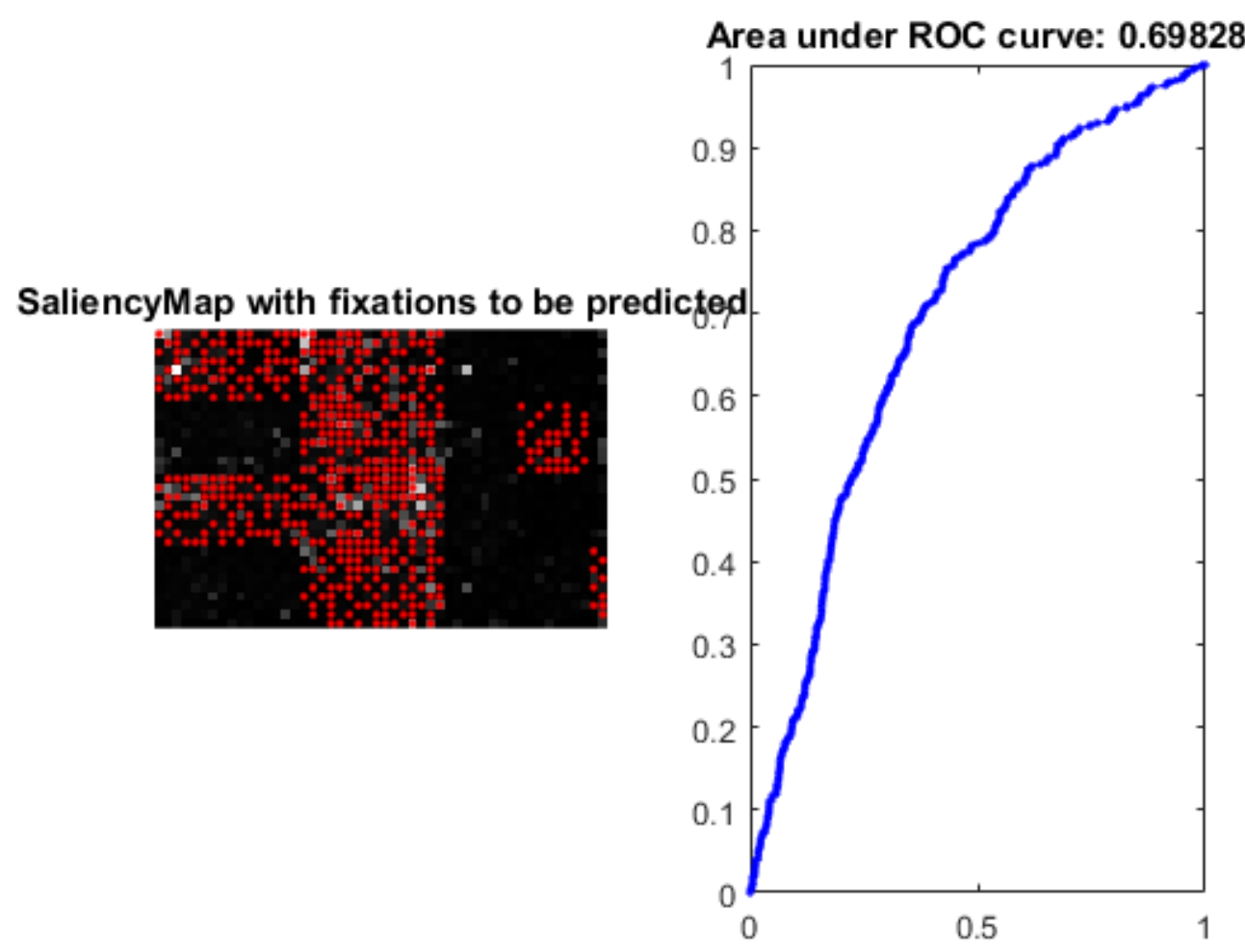

Figure 6. The maximal score of the generated maps on the AUC-Judd test. FP are the False Positive variables, TP - the true positive variables. 
Table 1. The general parameters of the approach.

\begin{tabular}{|l|l|}
\hline Input & $\begin{array}{l}\text { Real images (feed forward) + human fixation maps } \\
\text { (feedback for softmax) }\end{array}$ \\
\hline Supervised & Yes (early layers remained unsupervised) \\
\hline Filters required & No \\
\hline Toolbox & Matlab Neural Network Toolbox; Saliency Toolbox \\
\hline Spatial component algorithm & Autoencoders + Softmax \\
\hline Temporal component algorithm & Leaky integrate-and-fire \\
\hline
\end{tabular}


Table 2. Parameters of the LIF model.

\begin{tabular}{|l|l|l|}
\hline Parameter & Description & Value \\
\hline timeStep & time step for integration $\left(1 * 10^{-3} \mathrm{~ms}\right)$ & 0.001 seconds \\
\hline Eleak & leak potential & 0 volts \\
\hline Eexc & potential for excitatory channels & $100 \mathrm{e}-3$ volts \\
\hline Einh & potential for inhibitory channels & $-20 \mathrm{e}-3$ volts \\
\hline Gleak & leak conductivity & $1 \mathrm{e}-8$ Siemens \\
\hline Gexc & conductivity of excitatory channels & 0 Siemens \\
\hline Ginh & conductivity of inhibitory channels & 0 Siemens \\
\hline GinhDecay & time constant for decay of inhibitory conductivity & 1 Siemens \\
\hline Ginput & input conductivity & $5 \mathrm{e}-8$ Siemens \\
\hline Vthresh & threshold potential for firing & 0.001 volts \\
\hline C & capacity & $1 \mathrm{e}-9$ farads \\
\hline V & current membrane potential & 0 amperes \\
\hline I & current input current & . \\
\hline
\end{tabular}




\section{References}

1. Basheer, I. A., \& Hajmeer, M. (2000). Artificial neural networks: fundamentals, computing, design, and application. Journal of microbiological methods, 43(1), 3-31.

2. Bengio, Y. (2009). Learning deep architectures for AI. Foundations and trends ${ }^{\circledR}$ in Machine Learning, 2(1), 1-127.

3. Broadbent, D. (1987). Simple models for experimentable situations. In P. Morris (Ed.), Modelling cognition (p. 169185). John Wiley \& Sons.

4. Bylinskii, Z., Judd, T., Borji, A., Itti, L., Durand, F., Oliva, A., \& Torralba, A. (2015). Mit saliency benchmark.

5. Bylinskii, Z., Judd, T., Oliva, A., Torralba, A., \& Durand, F. (2016). What do different evaluation metrics tell us about saliency models?, 1-24. Retrieved from http://arxiv.org/abs/1604.03605

6. Das, S., \& Pedroni, B. (n.d.). Implementation of a Restricted Boltzmann Machine in a Spiking $\begin{array}{llll}\text { Neural Network. } & \text { Isn.Ucsd.Edu. } & \text { Retrieved } & \text { from }\end{array}$ http://www.isn.ucsd.edu/courses/bggn260/2012/reports/Das_Pedroni.pdf

7. Ferri, C., Hernández-Orallo, J., \& Flach, P. A. (2011). A coherent interpretation of AUC as a measure of aggregated classification performance. In Proceedings of the 28th International Conference on Machine Learning (ICML-11) (pp. 657-664).

8. Huang, X., Shen, C., Boix, X., \& Zhao, Q. (2015). Salicon: Reducing the semantic gap in saliency prediction by adapting deep neural networks. In Proceedings of the IEEE International Conference on Computer Vision (pp. 262270).

9. Itti, L., \& Koch, C. (2000). A saliency-based search mechanism for overt and covert shifts of visual attention. Vision Research, 40(10-12), 1489-1506. https://doi.org/10.1016/S0042-6989(99)00163-7

10. Judd, T., Durand, F., \& Torralba, A. (2012). A benchmark of computational models of saliency to predict human fixations.

11. Kummerer, M., Wallis, T. S., Gatys, L. A., \& Bethge, M. (2017). Understanding low-and high-level contributions to fixation prediction. In Proceedings of the IEEE International Conference on Computer Vision (pp. 4789-4798).

12. LeCun, Y., Bengio, Y., \& Hinton, G. (2015). Deep learning. nature, 521(7553), 436. 
13. Kachurka, V., Madani, K., Sabourin, C., \& Golovko, V. (2015, June). From human eye fixation to human-like autonomous artificial vision. In International Work-Conference on Artificial Neural Networks (pp. 171-184). Springer, Cham.

14. Krasovskaya, S., \& MacInnes, W. J. (2019). Salience Models: A Computational Cognitive Neuroscience Review. Vision, 3(4), 56. doi:10.3390/vision3040056

15. Maass, W. (1997). Networks of spiking neurons: The third generation of neural network models. Neural Networks, 10(9), 1659-1671. https://doi.org/10.1016/S0893-6080(97)00011-7

16. MacInnes, W. J. (2017). Comparison of temporal models for spatial cuing. 5th annual Polish Eye tracking conference

17. Merzon, L., Malevich, T., Zhulikov, G., Krasovskaya, S., MacInnes, W.J. (2020). Temporal Limitations of the Standard Leaky Integrate and Fire Model. Brain Sciences, 10, 16. https://doi.org/10.3390/brainsci10010016

18. Purcell, B. A., Schall, J. D., Logan, G. D., \& Palmeri, T. J. (2012). From salience to saccades: multiple-alternative gated stochastic accumulator model of visual search. Journal of Neuroscience, 32(10), 3433-3446.

19. Ratcliff, R. (1978). A theory of memory retrieval. Psychological Review, 85(2), 59-108. https://doi.org/10.1037/0033-295X.85.2.59

20. Ratcliff, R., \& McKoon, G. (2008). The Diffusion Decision Model: Theory and Data for TwoChoice Decision Tasks. Neural Computation, 20(4), 873-922. https://doi.org/10.1162/neco.2008.12-06-

420

21. Riche, N., Duvinage, M., Mancas, M., Gosselin, B., \& Dutoit, T. (2013, December). Saliency and human fixations: state-of-the-art and study of comparison metrics. In Computer Vision (ICCV), 2013 IEEE International Conference on (pp. 1153-1160). IEEE.

22. Russell, B. C., Torralba, A., Murphy, K. P., \& Freeman, W. T. (2008). LabelMe: a database and web-based tool for image annotation. International journal of computer vision, 77(1-3), 157-173.

23. Treisman, A. M., \& Gelade, G. (1980). A feature-integration theory of attention. Cognitive Psychology, 12(1), 97136. https://doi.org/10.1016/0010-0285(80)90005-5

24. Usher, M., \& McClelland, J. L. (2001). The time course of perceptual choice: The leaky, competing accumulator model. Psychological Review, 108(3), 550-592. https://doi.org/10.1037/0033295X.108.3.550

25. Walther, D., \& Koch, C. (2006). Modeling attention to salient proto-objects. Neural networks, 19(9), 1395-1407. 
26. Wolfe, J. M. (1994). Guided Search 2.0 A revised model of visual search. Psychonomic Bulletin \& Review, 1(2), 202-238. https://doi.org/10.3758/BF03200774

27. Wolfe, J. M., \& Gancarz, G. (1997). Guided Search 3.0. In Basic and clinical applications of vision science (pp. 189192). Springer, Dordrecht. 